\title{
EFICIENCIA DE LA Ammophila arenaria EN ATRAPAR SEDIMENTOS TRANSPORTADOS POR EL
} VIENTO.

\author{
Carlos Tavares Corrêa ${ }^{1}$
}

\section{Resumen}

El objetivo del presente estudio fue evaluar la eficiencia de la Ammophila arenaria en atrapar sedimentos en dunas litorales. Con este fin, se efectuó la comparación entre un sector con densa cobertura vegetal y otro sin vegetación, en las dunas litorales de costa central de Chile ( $\left.38^{\circ} 05^{\prime} \mathrm{S}\right)$. Para determinar las tasas de transporte eólico de sedimentos se utilizaron trampas verticales dispuestas a lo largo de perfiles en el inicio de la formación de dunas, en la base y en la cima de las dunas litorales. Los resultados mostraron que los granos de arena se quedan atrapados por la vegetación en la cara de barlovento y una cantidad muy insignificante $(3 \%)$ avanza por detrás de las dunas. Por otra parte, en sectores sin vegetación como en las cubetas de deflación, la capacidad de transporte aumentó exponencialmente en la cima, alcanzando valores de hasta 447\% con respecto a aquellos encontrados en la base de las dunas. Se comprueba la gran capacidad de retención de sedimentos que posee la Ammophila arenaria y se considera los sectores de dunas litorales con densas coberturas de Ammophila arenaria como verdaderas trampas naturales para la evaluación de tasas de transporte eólico de arenas.

Palabras clave: Ammophila arenaria, cubetas de deflación, Chile, dunas litorales, manejo de dunas, procesos eólicos, trampas de sedimento.

\begin{abstract}
The object of this paper was to evaluate the efficiency of the Ammophila arenaria in catching sediments. It was made by comparing sectors with dense vegetation cover and bare dunes (blowout), on foredunes at the central coast of Chile $\left(38^{\circ} 05^{\prime} \mathrm{S}\right)$. Vertical sand traps were placed across transects at the beginning of the dune formation, and at the base and the top of the foredune, in order to determine the aeolian sediment transport rates. The results showed that in the windward slope with dense vegetation the sand grains were trapped and an insignificant amount $(3 \%)$ advanced behind the dunes. On the other hand, on the blowout, the sand windblown increased exponentially to the top reaching values up to $447 \%$ with respect to those found at the base of the blowout. The great capacity of the Ammophila arenaria in catching sediments is verified and it considers that foredunes with dense covers of Ammophila arenaria are like true natural traps for the evaluation of aeolian sand transport rates.
\end{abstract}

Key words: aeolian processes, Ammophila arenaria, blowout, Chile, Dune Management, Foredune, sand traps.

\section{Introducción}

Las dunas litorales son formas de acumulación de arena por el viento que ocupan una franja relativamente angosta junto a la playa, y donde la vegetación ejerce un importante papel en la retención de sedimentos. La influencia primaria de la vegetación es la de modificar la velocidad del viento junto al suelo, de modo que se reduce la tensión sobre los sedimentos (Hesp, 1981). Así, la vegetación se constituye en un elemento de rugosidad superficial, cuya influencia aumenta con el aumento de la altura y densidad (Thomas, 1975). Según Jackson (1981), el nivel en que actúa la tensión superficial del viento se denomina el límite aerodinámico, que equivale al nivel promedio de la rugosidad superficial. Debajo de este límite, la influencia del viento es nula y los sedimentos se depositan. Donde la vegetación está ausente, la superficie arenosa es la que controla el límite aerodinámico.

La Ammophila arenaria es una especie psamófila reconocida como constructora de dunas, debido a su eficiencia en atrapar y estabilizar la arena. Estos atributos han motivado su introducción en los litorales templados de todos los continentes, donde se pretendió fijar arenas móviles (Paskoff, 1992), como por ejemplo, en la costa central de Chile. Allí, el proceso de estabilización de los campos de dunas en la costa tenía como propósito favorecer a la actividad forestal (Gormáz, 1974). El manejo consistió en la formación de dunas litorales con la Ammophila arenaria para retener las arenas que ingresan desde la playa.

Tavares Corrêa (1995) estimó la tasa de ingreso de arenas desde la playa a partir del análisis volumétrico

\footnotetext{
${ }^{1}$ Pontificia Universidad Católica del Perú, Av. Universitaria s/n. Cuadra 18, San Miguel, Lima 32, PERÚ. Telefax 51-1-2618867. Correo electrónico: ctavare@pucp.edu.pe
} 
de estas dunas litorales manejadas en la costa central de Chile. Las tasas encontradas, entre 8 y $25 \mathrm{~m}^{3} \mathrm{~m}^{-1}$ año ${ }^{-1}$, fueron estimadas considerándose una eficiencia de $100 \%$ en la retención de las arenas por parte de la vegetación. Estudios de Carter \& Wilson (1990) en la costa de Irlanda del Norte, estimaron que una duna litoral estabilizada por Ammophila arenaria captura entre $50 \%$ y $70 \%$ de la arena proveniente de la playa. En este sentido, Sherman (1995) considera que el volumen de la duna litoral solamente representa un valor mínimo de la tasa de transporte eólico de sedimentos desde la playa. Con el propósito de validar los datos obtenidos por Tavares Corrêa (1995), se evalúa la eficiencia de la Ammophila arenaria en la retención de sedimentos en las dunas litorales de Chile Central.

\section{Materiales y métodos}

\section{Área de estudio}

El área de estudio (Figura 1) se ubica en la zona costera de la provincia de Arauco, VIII Región, en la localidad de Paicavi (38 05' S). Comprende el sector central de una amplia llanura de $60 \mathrm{Km}$ de extensión y $6 \mathrm{Km}$ de ancho en promedio, modelada por dunas. (Tavares Corrêa, 1996). En esta zona el clima es de costa occidental con influencia mediterránea (Fuenzalida, 1971), donde el régimen pluviométrico es invernal con un promedio anual de $1.202 \pm 300 \mathrm{~mm}$, según los datos de la estación meteorológica de la Isla Mocha (1927-1970). Los vientos dominantes son de $\mathrm{W}$ a $\mathrm{N}$ entre mayo y agosto, y de S a SW en los meses restantes. Los vientos de $\mathrm{S}$ y $\mathrm{SW}$ son los más efectivos para el transporte eólico ya que los de $\mathrm{W}$ a $\mathrm{N}$ predominan en los meses de mayor precipitación.

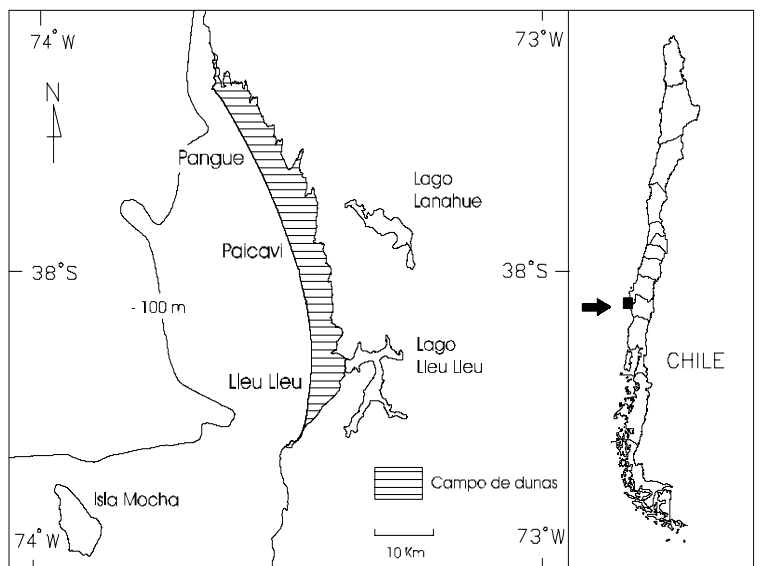

Figura 1. Localización del campo de dunas de la Provincia de Arauco.

En el ambiente marino, donde las pendientes son inferiores a $2^{\circ}$ entre la playa y la isóbata de $100 \mathrm{~m}$, dominan las olas de mar de leva de SW con energía alta a moderada (Araya - Vergara, 1985). En este sector costero, el estado morfodinámico predominante es el disipativo, caracterizado por bajas pendientes, playa amplia compuesta de arenas finas y la presencia de extensos campos de dunas a partir de la playa posterior. El gran potencial eólico en el área de estudio, es el principal factor limitante al crecimiento de vegetación pionera en la playa y a la formación de una duna litoral. Así, en el ambiente eólico predomina la duna transversa que se forma a partir de la pleamar debido a la acción de los fuertes vientos de SW.

La introducción de especies vegetales como la Ammophila arenaria para el control de las dunas en el sector, ha provocado cambios drásticos en la morfología de la franja costera con la formación de dunas litorales. Los primeros trabajos de manejo en el área de estudio datan de 1966. Según Gormáz (1974), lo que fue el primer paso para la estabilización del campo dunario con el propósito de prepararlo para la actividad forestal. Un estudio más reciente, (Tavares Corrêa, 1996) ha identificado que pasado 30 años, estas dunas litorales formadas por la Ammophila arenaria se mantienen conservadas o parcialmente deterioradas en el $50 \%$ de la línea litoral, aproximadamente. Sin embargo, en los sectores donde éstas no sufrieron un mantenimiento continuo, las dunas litorales fueron casi completamente destruidas por cubetas de deflación (blowouts), quedando en el paisaje solamente algunos montículos remanentes cubiertos con Ammophila arenaria.

\section{Métodos}

Se utilizó trampas verticales con el propósito de evaluar la eficiencia de la Ammophila arenaria en atrapar los sedimentos transportados por el viento. Para esto, se comparó un sector de dunas litorales que presentaba densa cobertura vegetal, con otro donde la poca densidad permitió la formación de cubetas de deflación. Con este propósito se levantó un perfil transversal en cada sector, mediante el uso de clinómetro y huincha métrica.

Este experimento consistió en la utilización de 3 trampas verticales de sedimentos, ubicadas a lo largo de cada perfil en el inicio de la formación de dunas en la playa posterior, en la base y en la cima de las dunas litorales (Figuras 2 y 3). Los muestreos fueron realizados entre 5 y 6 de enero de 1994, en el perfil con cobertura vegetal y en la cubeta de deflación, respectivamente, cuando se consideró tiempos de exposición para las trampas de 15, 30 y hasta 60 minutos, de acuerdo con la fuerza del viento y el volumen de arena atrapado. Se analizó los resultados relacionando la cantidad de arena capturada, expresada en $\mathrm{g} \mathrm{m}^{-1} \mathrm{~h}^{-1}$, entre las trampas de la base y la cima de la duna litoral con respecto a la del inicio de la formación de dunas, en posición más cercana a la playa.

La trampa vertical utilizada es una adaptación del modelo original de Leatherman (1978). Consiste en un tubo de PVC de 1 metro de largo, diámetro de 0.05 
metros y aberturas de 0.010 y 0.025 metros de ancho en su longitud, posterior y anterior. Para evitar la pérdida de granos y permitir el libre paso del flujo de aire a través de la trampa, se utilizó una malla de 62 micrones en la abertura anterior evitando así, interferencias en el flujo de aire y la consecuente pérdida de eficiencia en capturar los granos de arena (Sarre, 1988).

\section{Resultados y discusión}

Como forma de evaluar la eficiencia de la Ammophila arenaria en retener los sedimentos transportados por el viento, se efectuó la comparación entre dos sectores de la duna litoral: un sector cubierto con esta especie vegetal y otro comprendido por una cubeta de deflación. En las figuras 2 y 3 se representa los perfiles transversales a estos sectores y, además, la ubicación de las trampas de sedimentos.
El perfil de la Figura 2 representa un sector de la duna litoral protegido por densa cobertura de Ammophila arenaria. La playa tiene pendientes de $1^{\circ}$ a $3^{\circ}$ y ancho que varia entre 30 y $50 \mathrm{~m}$, debido a la amplitud de marea. El inicio de la formación de dunas en la playa posterior, se da en una franja entre 50 y 70 $\mathrm{m}$, cuya variación se debe a la fluctuación de la marea que controla el ingreso de arena al sistema. En esta unidad, se forman dunas barjanoides coalescentes con celeridad de 5 a $10 \mathrm{~m}$ año ${ }^{-1}$ y las depresiones están cubiertas de conchas. Estas dunas se incorporan a las dunas litorales a partir de los $140 \mathrm{~m}$. Las dunas litorales presentan altitud máxima de $6,89 \mathrm{~m}$, ancho de $50 \mathrm{~m}$ y cobertura vegetal que varia entre $50 \%$ y $90 \%$, a partir del punto medio de la cara de barlovento hasta su parte posterior, lo que permite el desarrollo de pendientes superiores a $20^{\circ}$ y $30^{\circ}$, respectivamente.

Por otra parte, el perfil de la Figura 3 representa

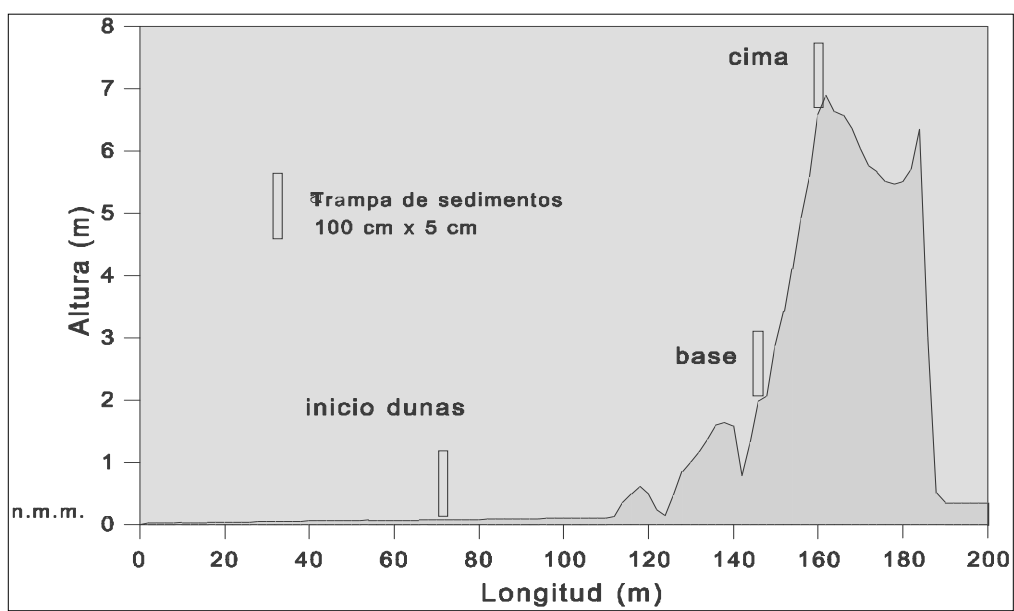

Figura 2. Perfil trasversal a la playa y duna litoral cubieerta con ammophilia arenaria y la ubicación de las trampas de sedimentos. de deflación. una cubeta de deflación que corta transversalmente las dunas litorales. Éste se caracteriza como un corredor de deflación formado en sectores con menor densidad de Ammophila arenaria. Allí, la playa presenta pendientes de $1^{\circ}$ a $3^{\circ}$ y ancho de 20 a $40 \mathrm{~m}$. Las características de playa posterior son idénticas al perfil anterior, siendo que las dunas barjanes coalescentes se incorporan al corredor de deflación a partir de los $80 \mathrm{~m}$. Las pendientes en su interior son inferiores a los $20^{\circ}$, con una altitud máxima de 4,87 $\mathrm{m}$ y ancho de $70 \mathrm{~m}$. Una densa cobertura de Ammophila arenaria estabiliza la cima de las paredes laterales de la cubeta

La Figura 4 contiene los resultados obtenidos con

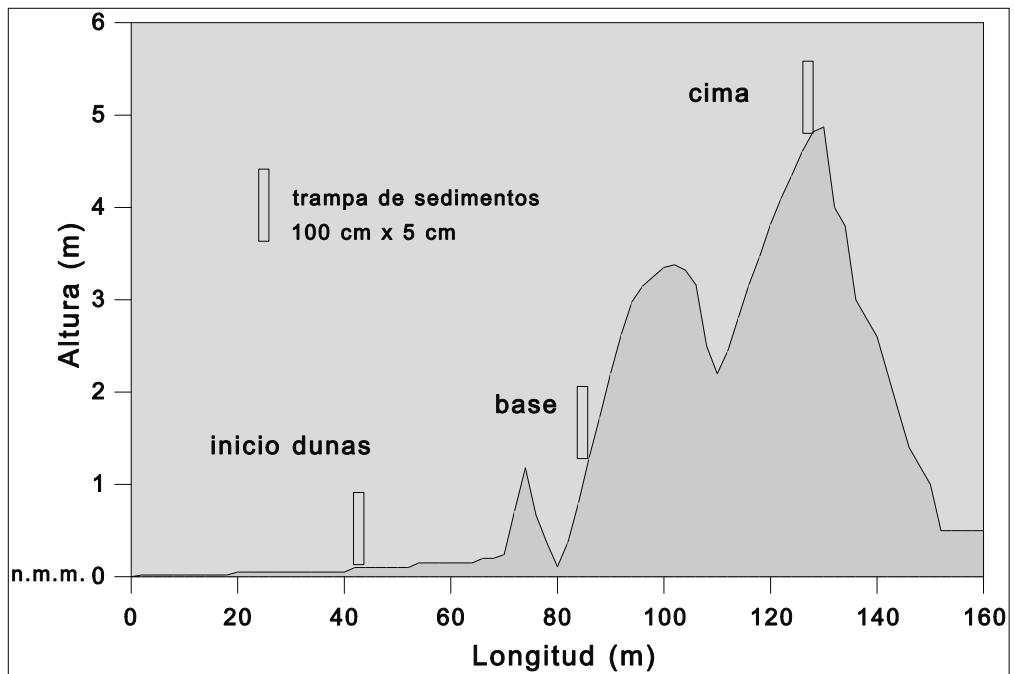
las trampas de sedimentos ubicadas en el inicio de la formación de dunas, en la base y en la cima de las dunas litorales cubiertas con vegetación. En los cinco muestreos realizados en este sector, se ha atrapado una mayor cantidad de arena en la base de la duna, con relación a la playa, seguido de una brusca reducción en la cima. En la Tabla 1 se observa que la cantidad de arena atrapada en la base de las dunas litorales es en promedio 4,7 veces mayor que la cantidad de arena capturada al inicio de la formación de dunas y que, en la cima, este valor disminuye a un promedio de 0,129 veces, con relación al inicio.

Por otra parte, la Figura 5 muestra

Figura 3. Perfil transversal a la playa y duna litoral en un sector con desarrollo de cubetas de deflación y ubicación de las trampas de sedimentos. los resultados alcanzados en la cubeta de deflación. A partir de 12 muestreos se ha identificado un aumento progresivo de la cantidad de arena atrapada tanto en la base como en la cima de la cubeta de deflación. Este 
aumento, representado en la Tabla 2, es del orden de 45,4 veces en la base y de 203,2 veces en la cima, en promedio, con respecto al inicio de las dunas, en la playa posterior. avanza por detrás de las dunas. Por otra parte, en el sector libre de vegetación la cantidad de arena atrapada aumenta significativamente hacia la parte superior de la cubeta de deflación $(447 \%)$. Esto ocurre

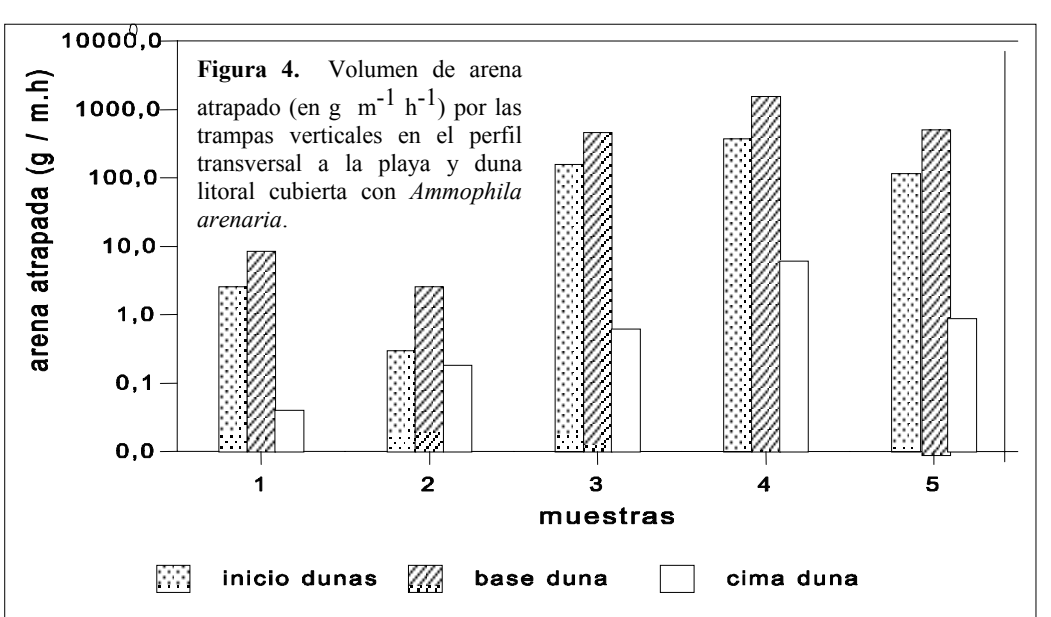

porque dicho transporte aumenta exponencialmente sobre una ladera, alcanzando valores máximos en la parte superior. En ausencia de vegetación, el perfil sufrirá un mayor arrastre de arenas en la cima, y grandes cantidades de sedimentos se movilizarán detrás de la duna. En éstos sectores no protegidos, el perfil de la duna tiende a suavizarse y a ensancharse, pues la cantidad de arena que abastece la cima de la ladera es inferior a aquélla que la abandona, provocando un efecto semejante al control morfológico de dunas transversales descritos por Lancaster (1985) y Watson (1987).

En este sentido, los sectores de las dunas litorales que presentan densas coberturas de Ammophila arenaria se constituyen en verdaderas trampas naturales para la evaluación de tasas de transporte eólico de arenas. La utilización de este indicador en los estudios de la dinámica eólica en los campos de dunas de la costa Chilena, permitieron a que Tavares Corrêa $(1995,1996)$ allí pudiese alcanzar resultados satisfactorios que, según Sherman (1995) pueden representar un potencial mínimo para el área de estudio.

\section{Conclusiones}

Se evaluó la eficiencia de Ammophila arenaria en atrapar sedimentos, a partir de lo cual se constató su gran capacidad de retención. En una ladera de barlovento de las dunas litorales donde la cobertura de Ammophila arenaria es densa, la capacidad de retención de sedimentos alcanza hasta el $97 \%$ de eficiencia. Por otra parte, en sectores sin vegetación como en las cubetas de deflación, la capacidad de transporte aumenta exponencialmente en la cima, alcanzado valores de hasta $447 \%$ con respecto a los valores encontrados en la base de las dunas. Por lo tanto, se puede considerar los sectores de dunas litorales con densas coberturas de Ammophila arenaria como verdaderas trampas naturales para la evaluación de tasas de transporte eólico de arenas.

\section{Agradecimientos}

Este estudio fue realizado gracias al apoyo financiero de la Dirección de Investigación de la Universidad de Concepción (Proyecto n94.310.07-6) y la infraestructura del Centro EULA - Chile. De igual manera se agradece a Marco Cisternas y Arturo Caro por el inestimable apoyo durante los trabajos de campo y al Dr. Hildegardo Córdova por los 
comentarios y sugerencias.

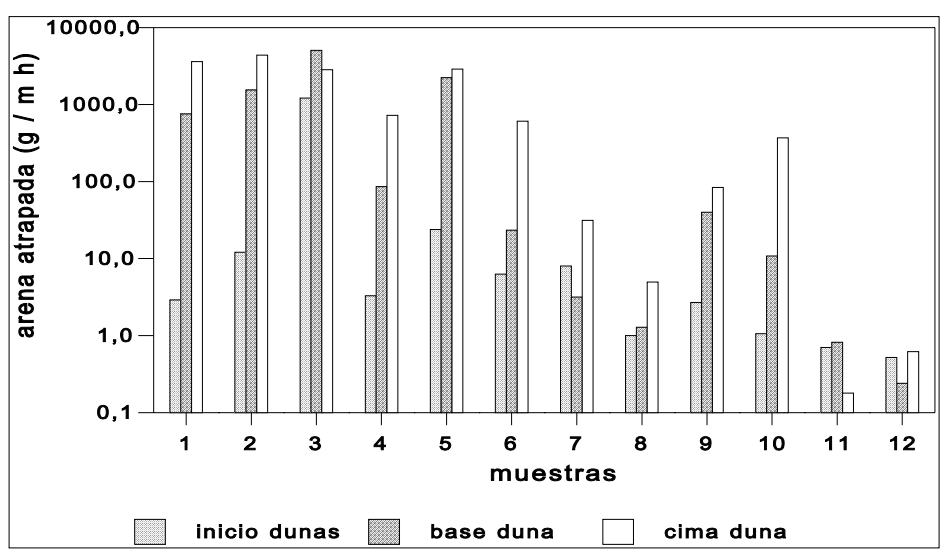

sand transport on windward flanks of the desert sand dunes. Sedimentology. 32: 581-593.

Hesp P.A. 1981. The formation of shadow dunes. Journal of Sedimentary Petrology. 51(1): 101-111.

Jackson P. 1981. On the displacement height in the logarithimic velocity profile. Journal of Fluid Mechanic. 111: 15-25.

Leatherman S.P. 1978. A new aeolian sand trap design. Sedimentology. 25: 303-306.

Paskoff R. 1992. Las dunas del litoral. Mundo Científico. 8 (95): 958-965.

Sarre R. 1988. Evaluation of aeolian sand transport equations using intertidal-zone measurements, Saunton Sands, England. Sedimentology. 35: 671-679.

Figura 5. Volumen de arena atrapado (en $\left.\mathrm{g} \mathrm{m}^{-1} \mathrm{~h}^{-1}\right)$ por las trampas verticales en el perfil transversal a la playa y duna litoral en un sector con desarrollo de cubetas de deflación.

\section{Literatura citada}

Araya-Vergara J.F. 1985. Sediment supply and orphogenetic response on a high wave energy west coast. Z. Geomorph. N.F., Suppl. Bd. 57: 67-79.

Carter R.W:G. \& Wilson, P. 1990. The geomorphological, ecological and pedological development of coastal foredunes at Magilligan Point, Northern Ireland. Páginas 129 - 157 en: K. F. Nordstrom, N. Psuty \& R.W.G. Carter (editores) Coastal dunes: form and process. John Wiley \& Sons, London.

Fuenzalida H. 1971. Climatología de Chile. Publ. Int. Fac. Cien. Fís. Mat., U. de Chile.

Gormáz M. 1974. Las dunas. Informe de la Corporación Nacional Forestal.

Lancaster N. 1985. Variations in wind velocity and
Sherman D.J. 1995. Problems of scale in the modeling and interpretation of coastal dunes. Marine Geology. 124: 339-349.

Tavares Corrêa C. 1996. Propuesta de uso del suelo en las dunas litorales de la provincia de Arauco, VIII Región, Chile. Tesis de Doctorado en Ciencias Ambientales, Centro EULA-Chile, Univ. de Concepción, Concepción, Chile.

Tavares Corrêa C. 1995. La importancia de la duna bordera artificial en la estimación del transporte 308.

Thomas Y.-F. 1975. Actions eoliennes en milieu littoral la Pointe de la Coubre. Memoires du Laboratoire de Geomorphologie de L'ecole Pratique des Hautes Etudes $n^{\circ} 29$.

Watson A. 1987. Discussion: variation in wind velocity and sand transport on the windward flanks of desert sand dunes. Sedimentology. 34: 511-520. eólico en la provincia de Arauco, Chile. Anales, XVI Congreso Chile de Geografía, Valdivia. : 303- 